\title{
Perturbation solutions of a mathematical model for determining the roles of Endothelial, pericyte and macrophage cells in the capillary
}

\author{
Serdal Pamuk* and Melike Keles \\ Department of Mathematics, University of Kocaeli, Kocaeli, Turkey
}

Received: 12 December 2019, Accepted: 3 February 2020

Published online: 18 February 2020.

\begin{abstract}
The purpose of this work is to obtain the regular perturbation solutions of a mathematical model for capillary formation in tumor angiogenesis. The model we study here was originally presented in [Levine HA, Sleeman BD and Nilsen-Hamilton M., 2000]. The regular perturbation method is a well-known and highly effective method to obtain the solutions of coupled non-linear differential equations. In fact, a few terms of the perturbation series obtained by this method are good enough to see the structure of the solutions of the model. These solutions govern the movement of the certain cells, namely endothelial, macrophage and pericyte cells, in the capillary which are necessary for the initiation of tumor angiogenesis. Our MATLAB-generated figures show that our numerical simulations are in good agreement with the biological facts about the tumor angiogenesis. Even though computing the terms of the regular perturbation series are kind of tedious, more stable and accurate solutions of the model can be obtained by adding new terms to the series.
\end{abstract}

Keywords: Regular Perturbation Solution, Tumor Angiogenesis, Endothelial Cell, Pericyte Cell, Macrophage Cell.

\section{Introduction}

Angiogenesis is the formation of new blood vessels from pre-existing vessels in a tissue. It is necessary for growth and development of tumors and wound healing. It is also known to occur in three consecutive steps [2]. First, the endothelial cells (EC) lining the vascular basal lamina (BL) (or basement membrane) degrade this membrane. Second, the EC migrate and proliferate (via mitosis) into the extra cellular matrix (ECM). Finally, capillary loops form.

Fibronectin, one of the major components of the ECM, is large adhesive glycoproteins consisting of two fibrous polypeptides particularly abundant in plasma, connective tissue matrices, and BL [3]. It is also known to increase EC adhesion to collagen and is produced by EC [4]. Fibronectin serves as a specific pathway for cell proliferation, differentiation and cell migration.

EC, as argued in [5,6], are stimulated by a tumor angiogenic factor for angiogenesis to occur. Furthermore, the active enzyme stimulates the migration of EC [7]. The long time tendency of endothelial cells, which are stimulated, is towards the transition probability density function (TPDF) [8] of active enzyme and fibronectin (see [9] for mathematical proof of this). Endothelial cell migration and proliferation also occur during endothelial repair in situ; the ability to penetrate the vascular basement membrane, on the other hand, is an aspect of endothelial cell behavior uniquely expressed during angiogenesis [10]. We have also considered pericyte cells (PC) and macrophage cells (MC) to examine their role in angiogenesis.

PC, which form a periendothelial cellular network within the basal lamina, are intimately entailed in the regulation of the 
proliferation of endothelial cells. As stated in [15] the term pericyte was initially used to describe cells that were found adjacent to capillaries. These cell types have a small, oval cell body. Also, inflammatory cells, such as macrophages, which mathematical modeling of tumor-induced angiogenesis contribute to the production of angiogenic factors in an environment already enriched with angiogenic stimuli may also attract tumor cells [15]. Macrophages are scavenger cells obtained from monocyte blood cells released from the bone marrow.

There have been many mathematical models related to tumor angiogenesis in one or more space dimensions [11-14]. In fact, in [11] a critical review of selected topics related to the modeling of cancer onset, evolution and growth, with the aim of illustrating, to a wide applied mathematical readership, some of the novel mathematical problems in the field is presented. In [12] a review and critical analysis of the asymptotic limit methods focused on the derivation of macroscopic equations for a class of equations modeling complex multicellular systems by methods of the kinetic theory for active particles is proposed. Also, in [13] the authors deal with the derivation of macroscopic equations for a class of nonlinear equations modeling complex multicellular systems, and in [14] the authors present a continuous model for three early stage events in angiogenesis: initiation, sprout extension and vessel maturation. On the other hand, in [9, 22], mathematical analyses of a mathematical model for capillary formation in tumor angiogenesis are presented in one and two space dimensions, respectively.

In [27] the authors investigate the impact of heat transfer on the magnetohydrody amic peristaltically induced motion in a channel through porous medium. The continuity, momentum, and energy equations have been used to represent the flow in a closed form, and these non-linear governing equations are solved analytically by employing the perturbation method. Also, in [28] peristaltic motion induced by sinusoidal traveling wave of incompressible, electrically conducting Maxwell fluid in the porous walls of a two dimensional channel through a porous medium is studied in the presence of a constant magnetic field, and in [29] the peristaltic motion of a compressible and electrically conducting Jeffrey fluid induced by a surface acoustic wave in a confined parallel plane microchannel through a porous medium is analytically investigated. The problems in $[28,29]$ are analyzed using a perturbation expansion in terms of small amplitude ratio. Lastly, in [30] the authors concern with theoretically investigating the pulsatile flow of a fluid with suspended particles in a flow driven by peristaltic waves that deform the wall of a small blood artery in the shape of traveling sinusoidal waves with constant velocity, and in [31] the authors examine peristaltic motion induced by a surface acoustic wave of a viscous, compressible and electrically conducting Maxwell fluid in a confined parallel-plane microchannel through a porous medium in the presence of a constant magnetic field.

The rest of the paper is organized as follows. First, we define the regular perturbation method used in this work. Second, the model originally presented in [1] is presented briefly. Third, we apply the regular perturbation method to our equations, and obtain the series solutions of them. Finally, we close the paper by providing a numerical example and the conclusions section.

\section{Regular perturbation method}

Regular perturbation theory deals with those problems for which a small change of perturbation parameter induces a small change in the solution. In regularly perturbed problems, the small parameter is present in the term other than the one involving the highest order derivative [16].

A basic feature of all regular perturbation problems is that the exact solution for small but non zero $\varepsilon$ smoothly approaches the unperturbed or zeroth-order solution $\varepsilon \rightarrow 0$ [17]. A perturbative solution is constructed as a series of powers of $\varepsilon$ :

$$
y(x, \varepsilon)=y_{0}(x)+\varepsilon y_{1}(x)+\varepsilon^{2} y_{2}(x)+\ldots
$$


This series is called a perturbation series [17]. Here, $y_{0}(x)$ is the solution to the simplified form of the original problem, (i.e. the zeroth-order solution) and $y_{1}(x), y_{2}(x), \ldots$ are the solutions of the equations obtained by substituting the power series into the original problem and expanding all quantities in a power series of $\varepsilon$ and collecting terms with same powers of $\varepsilon$ and equating them to zero [23-25]. If $\varepsilon$ is very small, we expect that $y(x)$ will be well approximated by only a few terms of the perturbation series [17].

\section{The model}

In the cartesian coordinate system we assume a capillary wall of the length $l_{2}$ microns located in the interval $\left[0, l_{2}\right]$ on the $y$ axis with a tumor source located someplace along the line $x=l_{1}$. We imagine the problem of tumor vascularization as shown in Figure 1. By rescaling the $x$ by $x / l_{1}$ and $y$ by $y / l_{2}$, this rectangle becomes a unit square. Hence, we now get $0 \leq x, y \leq 1$. Fundamentally, the problem comprise two parts: The first part is the dynamics on the y axis i.e., in the capillary (1D problem), The second part is the dynamics in the unit square i.e., in the ECM (2D problem). We combine those two dynamics via some boundary conditions (see $[1,18]$ for details). A regular perturbation solution of the model for EC, PC and MC density equations in ECM is the focus on this paper.

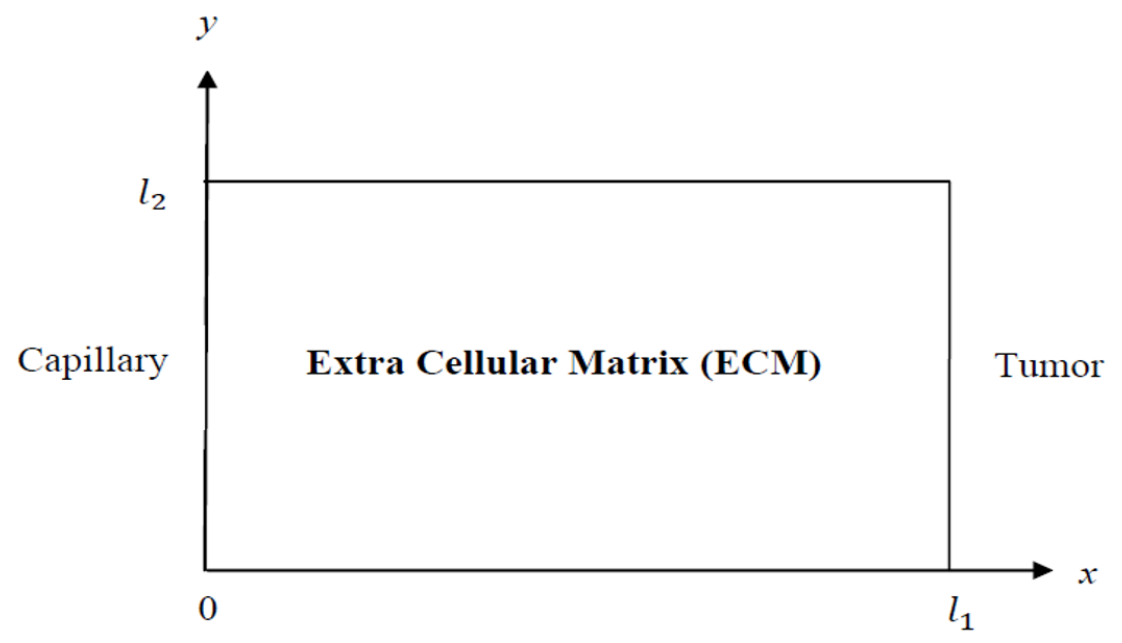

Fig. 1: Extra Cellular Matrix (ECM).

We consider the following notation for the functions:

$\mathrm{u}=$ concentration of chemotactic agent,

$\mathrm{v}=$ concentration of angiogenic factor,

$\mathrm{c}=$ concentration of proteolytic enzyme,

$\mathrm{f}=$ density of fibronectin,

$\eta=$ endothelial cell density,

$\sigma=$ perictye cell density,

$\mathrm{m}=$ macrophage cell density.

In the following mechanisms we use the law of mass action for concentrations of the chemotactic agent, angiogenic factor, proteolytic enzyme and fibronectin while we use reinforced random walk ideas [19] for the dynamics of EC, PC and MC. 
Let $\mathrm{U}, R_{M a}$ and $\left[R_{M a} U\right]$ be chemotactic attractant for $\mathrm{MC}$, receptor on the MC and intermediate complex, respectively. Then, $\mathrm{U}$ and $R_{M a}$ react to generate the angiogenic factor $\mathrm{V}$ according to the following mechanism:

$$
U+R_{M a} \underset{k_{-1}}{\stackrel{k_{1}}{\longrightarrow}}\left[R_{M a} U\right] \stackrel{k_{2}}{\longrightarrow} V+R_{M a}
$$

Also, let $R_{E}$ be receptor on the EC wall. Then, $V$ and $R_{E}$ react to produce the proteolytic enzyme $\mathrm{C}$ as follows:

$$
V+R_{E} \underset{k_{-3}}{\stackrel{k_{3}}{\longrightarrow}}\left[R_{E} V\right] \stackrel{k_{4}}{\longrightarrow} C+R_{E}
$$

where $\left[R_{E} V\right]$ is the intermediate complex.

A reaction mechanism for the decay of fibronectin $(\mathrm{F})$ via protease is assumed as follows:

$$
C_{A}+F \stackrel{k_{7}}{\longrightarrow}\left[C_{A} F\right] \stackrel{k_{8}}{\longrightarrow} F^{\prime}+C_{A}
$$

where $C_{A}$ is active enzyme, $\left[C_{A} F\right]$ is the intermediate complex and $F^{\prime}$ is a new product obtained by the degradation of $\mathrm{F}$. First, if we apply the law of mass action to the equations (1)-(2) and then use the Michealis-Menten kinetics to equation (3) (see [1] for details), we obtain the following system of equations :

$$
\begin{gathered}
\frac{\partial u}{\partial t}=-\frac{\lambda_{2} u m}{1+v_{2} u}, \\
\frac{\partial v}{\partial t}=\frac{\lambda_{2} u m}{1+v_{2} u}-\frac{\lambda_{1} v \eta}{1+v_{1} v}, \\
\frac{\partial c}{\partial t}=\frac{\lambda_{1} v \eta}{1+v_{1} v}, \\
\frac{\partial f}{\partial t}=\beta(1-f) f \eta-\frac{\lambda_{4} c f}{1+v_{4} f},
\end{gathered}
$$

where $\lambda_{i}$ and $v_{i}(\mathrm{i}=1,2,4)$ are kinetic parameters, and $\beta$ is a positive constant.

Moreover, if we use the idea of reinforced random walk [19] we obtain the EC, PC and MC movement equations (see [1] for the derivation of the following equations), the equation governing the motion of EC is given as follows [1]:

$$
\frac{\partial \eta}{\partial t}=D_{1} \frac{\partial}{\partial x}\left(\eta \frac{\partial}{\partial x}\left(\ln \frac{\eta}{\tau_{1}}\right)\right)
$$

where $\tau_{1}$ is the so-called probability transition function and the positive constant $D_{1}$ is the EC diffusion coefficient in the capillary. We take $\tau_{1}=\tau_{1}(c, f)=\left(\frac{c+\alpha_{1}}{c+\alpha_{2}}\right)^{\gamma_{1}}\left(\frac{f+\beta_{1}}{f+\beta_{2}}\right)^{\gamma_{2}}$. Here $\alpha_{i}, \beta_{i}$ and $\gamma_{i}(i=1,2)$ are some positive constants such that $0<\alpha_{1} \gg l<\alpha_{2}$ and $\beta_{1}>1 \gg \beta_{2}>0$. Similarly, in [1] the governing equation for the motion of PC is of the form:

$$
\frac{\partial \sigma}{\partial t}=D_{2} \frac{\partial}{\partial x}\left(\sigma \frac{\partial}{\partial x}\left(\ln \frac{\sigma}{\tau_{2}}\right)\right),
$$

where $\tau_{2}$ is the so-called probability transition function and the positive constant $D_{2}$ is the PC diffusion coefficient in the capillary. We take $\tau_{2}=\tau_{2}(f)=\left(\frac{f+\alpha_{3}}{f+\alpha_{4}}\right)^{\gamma_{3}}$, where $0<\alpha_{3} \ll 1<\alpha_{4}$ and $\gamma_{3}$ are positive constants. Finally, the governing 
equation for the motion of MC is taken as follows [1]:

$$
\frac{\partial m}{\partial t}=D_{3} \frac{\partial}{\partial x}\left(m \frac{\partial}{\partial x}\left(\ln \frac{m}{\tau_{3}}\right)\right),
$$

where $\tau_{3}$ is the so-called probability transition function and the positive constant $D_{3}$ is the MC diffusion coefficient in the capillary. We take $\tau_{3}=\tau_{3}(u)=\left(\frac{u+\beta_{3}}{u+\beta_{4}}\right)^{\gamma_{4}}$, where $0<\beta_{3} \ll 1<\beta_{4}$ and $\gamma_{4}$ are positive constants.

We take $u(x, 0)=\theta(x), \mathrm{v}(\mathrm{x}, 0)=0, \mathrm{c}(\mathrm{x}, 0)=0, \mathrm{f}(\mathrm{x}, 0)=1, \eta(\mathrm{x}, 0)=\sigma(\mathrm{x}, 0)=\mathrm{m}(\mathrm{x}, 0)=1$ as the initial conditions and we take $\eta_{x}=$ $\sigma_{x}=m_{x}=0$ at $x=0,1$ as the boundary conditions. We choose the function $\theta(x)$ of the form $\theta(x)=(1-\cos (2 \pi x))$ and let $v_{1}=v_{2}=v_{4}=0$ in our computations.

We also take the transition probability functions $\tau_{1}, \tau_{2}$ and $\tau_{3}$ as follows. They are taken as the functions of $x$ only for simplicity: $\tau_{1}=C_{1} \exp \left(3\left(x-x^{2}\right)^{2}\right), \tau_{2}=C_{2} \exp \left(3\left(x-x^{2}\right)^{2}\right), \tau_{3}=C_{3} \exp \left(3\left(x-x^{2}\right)^{2}\right)$, where $C_{1}, C_{2}$ and $C_{3}$ are some arbitrary constants.

\section{Model analysis and solutions}

To solve our model using the regular perturbation method we perturb our model equations as follows:

$$
\begin{gathered}
\frac{\partial u}{\partial t}=-\lambda_{2} u(1+\varepsilon m), \\
\frac{\partial \mathrm{v}}{\partial \mathrm{t}}=\lambda_{2} \mathrm{u}(1+\varepsilon \mathrm{m})-\lambda_{1} v(1+\varepsilon \eta), \\
\frac{\partial c}{\partial t}=\lambda_{1} v(1+\varepsilon \eta) \\
\frac{\partial f}{\partial t}=\beta(1-\varepsilon f) \varepsilon f(1+\varepsilon \eta)-\lambda_{4} c(1-\varepsilon) . \\
\frac{\partial \eta}{\partial t}=D_{1}\left(\frac{\partial^{2} \eta}{\partial x^{2}}-\varepsilon A \frac{\partial \eta}{\partial x}-\varepsilon \eta \frac{\partial A}{\partial x}\right) \\
\frac{\partial \sigma}{\partial t}=D_{2}\left(\frac{\partial^{2} \sigma}{\partial x^{2}}-\varepsilon B \frac{\partial \sigma}{\partial x}-\varepsilon \sigma \frac{\partial B}{\partial x}\right), \\
\frac{\partial m}{\partial t}=D_{3}\left(\frac{\partial^{2} m}{\partial x^{2}}-\varepsilon C \frac{\partial m}{\partial x}-\varepsilon m \frac{\partial C}{\partial x}\right) .
\end{gathered}
$$

Here, $A=\frac{\tau_{1 x}}{\tau_{1}}, B=\frac{\tau_{2 x}}{\tau_{2}}$ and $C=\frac{\tau_{3 x}}{\tau_{3}}$. Let us assume that our variables $\mathrm{u}, \mathrm{v}, \mathrm{c}, \mathrm{f}, \eta, \sigma$ and $\mathrm{m}$ have the following perturbation series expansions, respectively.

$$
\begin{aligned}
& u(x, t, \varepsilon)=u_{0}(x, t)+\varepsilon u_{1}(x, t)+\varepsilon^{2} u_{2}(x, t)+\ldots \\
& v(x, t, \varepsilon)=v_{0}(x, t)+\varepsilon v_{1}(x, t)+\varepsilon^{2} v_{2}(x, t)+\ldots \\
& c(x, t, \varepsilon)=c_{0}(x, t)+\varepsilon c_{1}(x, t)+\varepsilon^{2} c_{2}(x, t)+\ldots \\
& f(x, t, \varepsilon)=f_{0}(x, t)+\varepsilon f_{1}(x, t)+\varepsilon^{2} f_{2}(x, t)+\ldots \\
& \eta(x, t, \varepsilon)=\eta_{0}(x, t)+\varepsilon \eta_{1}(x, t)+\varepsilon^{2} \eta_{2}(x, t)+\ldots
\end{aligned}
$$




$$
\begin{aligned}
& \sigma(x, t, \varepsilon)=\sigma_{0}(x, t)+\varepsilon \sigma_{1}(x, t)+\varepsilon^{2} \sigma_{2}(x, t)+\ldots \\
& m(x, t, \varepsilon)=m_{0}(x, t)+\varepsilon m_{1}(x, t)+\varepsilon^{2} m_{2}(x, t)+\ldots
\end{aligned}
$$

If we plug these expansions in equations (11)-(17), we obtain the following equations according to the powers of $\varepsilon$ :

$$
\begin{aligned}
& \varepsilon^{0}: u_{0 t}=-\lambda_{2} u_{0}, u_{0}(x, 0)=\theta(x), \\
& \varepsilon^{1}: u_{1 t}=-\lambda_{2}\left(u_{0} m_{0}+u_{1}\right), u_{1}(x, 0)=0 \text {, } \\
& \varepsilon^{0}: v_{0 t}=\lambda_{2} u_{0}-\lambda_{1} v_{0}, v_{0}(x, 0)=0, \\
& \varepsilon^{1}: v_{1 t}=\lambda_{2}\left(u_{0} m_{0}+u_{1}\right)-\lambda_{1}\left(v_{0} \eta_{0}+v_{1}\right), v_{1}(x, 0)=0 \text {, } \\
& \varepsilon^{0}: c_{0 t}=\lambda_{1} v_{0}, c_{0}(x, 0)=0, \\
& \varepsilon^{1}: c_{1 t}=\lambda_{1}\left(v_{1}+v_{0} \eta_{0}\right), c_{1}(x, 0)=0 \text {, } \\
& \varepsilon^{0}: f_{0_{t}}=-\lambda_{4} c_{0}, f_{0}(x, 0)=1 \text {, } \\
& \varepsilon^{1}: f_{1 t}=\beta f_{0}-\lambda_{4} c_{1}, f_{1}(x, 0)=0 \text {, } \\
& \varepsilon^{0}: \eta_{0_{t}}=D_{1} \eta_{0_{x x}}, \eta_{0}(x, 0)=1, \eta_{0_{x}}(0, t)=\eta_{0_{x}}(1, t)=0 \text {, } \\
& \varepsilon^{1}: \eta_{1_{t}}=D_{1}\left(\eta_{1_{x x}}-A \eta_{0_{x}}-A_{x} \eta_{0}\right), \eta_{1}(x, 0)=0, \eta_{1_{x}}(0, t)=\eta_{1_{x}}(1, t)=0 \text {, } \\
& \varepsilon^{0}: \sigma_{0 t}=D_{2} \sigma_{0 x x}, \sigma_{0}(x, 0)=1, \sigma_{0 x}(0, t)=\sigma_{0 x}(1, t)=0, \\
& \varepsilon^{1}: \sigma_{1 t}=D_{2}\left(\sigma_{1 x x}-B \sigma_{0 x}-B_{x} \sigma_{0}\right), \sigma_{1}(x, 0)=0, \sigma_{1 x}(0, t)=\sigma_{1 x}(1, t)=0 \text {, } \\
& \varepsilon^{0}: m_{0 t}=D_{3} m_{0 x x}, m_{0}(x, 0)=1, m_{0 x}(0, t)=m_{0 x}(1, t)=0 \\
& \varepsilon^{1}: m_{1 t}=D_{3}\left(m_{1 x x}-C m_{0 x}-C_{x} m_{0}\right), m_{1}(x, 0)=0, m_{1 x}(0, t)=m_{1 x}(1, t)=0
\end{aligned}
$$

Solving the initial value problems (19)-(22) give the following solutions:

$$
\begin{gathered}
u_{0}(x, t)=\theta(x) \exp \left(-\lambda_{2} t\right), \\
u_{1}(x, t)=-\lambda_{2} t \theta(x) \exp \left(-\lambda_{2} t\right), \\
v_{0}(x, t)=\frac{\lambda_{2}}{\lambda_{1}-\lambda_{2}} \theta(x)\left(\exp \left(-\lambda_{2} t\right)-\exp \left(-\lambda_{1} t\right)\right), \\
v_{1}(x, t)=\lambda_{2}^{2} \theta(x)\left(\frac{-t \exp \left(-\lambda_{2} t\right)}{\lambda_{1}-\lambda_{2}}+\frac{\exp \left(-\lambda_{1} t\right)-1}{\left(\lambda_{1}-\lambda_{2}\right)^{2}}\right)-\frac{\lambda_{1} \lambda_{2}}{\lambda_{1}-\lambda_{2}} \theta(x)\left(\frac{\exp \left(-\lambda_{2} t\right)-1}{\lambda_{1}-\lambda_{2}}-t \exp \left(-\lambda_{1} t\right)\right),
\end{gathered}
$$




$$
\begin{gathered}
c_{0}(x, t)=\frac{\lambda_{1} \lambda_{2}}{\lambda_{1}-\lambda_{2}} \theta(x)\left(\frac{1-\exp \left(-\lambda_{2} t\right)}{\lambda_{2}}+\frac{\exp \left(-\lambda_{1} t\right)-1}{\lambda_{1}}\right), \\
c_{1}(x, t)=\lambda_{1} \lambda_{2}{ }^{2} \theta(x)\left(\frac{\exp \left(-\lambda_{2} t\right)}{\lambda_{2}\left(\lambda_{1}-\lambda_{2}\right)}-\frac{\exp \left(-\lambda_{2} t\right)+1+\lambda_{2} t}{\lambda_{2}\left(\lambda_{1}-\lambda_{2}\right)^{2}}\right) \\
\quad-\frac{\lambda_{1} \lambda_{2}}{\lambda_{1}-\lambda_{2}} \theta(x)\left(-\frac{\exp \left(-\lambda_{2} t\right)+1+t}{\lambda_{1}-\lambda_{2}}+\frac{\exp \left(-\lambda_{1} t\right)-\exp \left(-\lambda_{1} t\right)-1}{\lambda_{1}}\right. \\
\left.\quad-\frac{\exp \left(-\lambda_{1} t\right)+1}{\lambda_{1}{ }^{2}}+\frac{\exp \left(-\lambda_{2} t\right)+1}{\lambda_{2}},\right) \cdot \\
f_{0}(x, t)=-\frac{\lambda_{1} \lambda_{2} \lambda_{4}}{\lambda_{1}-\lambda_{2}} \theta(x)\left(\frac{t}{\lambda_{2}}+\frac{\exp \left(-\lambda_{2} t\right)-1}{\lambda_{2}{ }^{2}}+\frac{-\exp \left(-\lambda_{1} t\right)+1}{\lambda_{1}{ }^{2}}-\frac{t}{\lambda_{1}}\right), \\
f_{1}(x, t)=-\frac{\lambda_{1} \lambda_{2} \lambda_{4} \beta}{\lambda_{1}-\lambda_{2}} \theta(x)\left(\frac{t^{2}}{2 \lambda_{2}}+\frac{-\exp \left(-\lambda_{2} t\right)+1}{\lambda_{2}{ }^{3}}-\frac{t^{2}}{\lambda_{2}{ }^{2}}+\frac{\exp \left(-\lambda_{1} t\right)-1}{\lambda_{1}{ }^{3}}-\frac{t}{\lambda_{1}{ }^{2}}\right) \\
-\lambda_{1} \lambda_{2}{ }^{2} \lambda_{4} \theta(x)\left(\frac{\exp \left(-\lambda_{2} t\right)}{\lambda_{2}\left(\lambda_{1}-\lambda_{2}\right)}+\frac{\exp \left(-\lambda_{2} t\right)-1}{\lambda_{2}{ }^{2}\left(\lambda_{1}-\lambda_{2}\right)}+\frac{-\exp \left(-\lambda_{2} t\right)+1}{\lambda_{2}\left(\lambda_{1}-\lambda_{2}\right)^{2}}-\frac{t}{\left.\lambda_{1}-\lambda_{2}\right)^{2}}\right) \\
+\frac{\lambda_{1} \lambda_{2} \lambda_{4}}{\lambda_{1}-\lambda_{2}} \theta(x)\left(\frac{-\exp \left(-\lambda_{2} t\right)+1-\lambda_{2} t}{\lambda_{2}\left(\lambda_{1}-\lambda_{2}\right)}+\frac{t \exp \left(-\lambda_{1} t\right)}{\lambda_{1}}+\frac{\exp \left(-\lambda_{1} t\right)-1}{\lambda_{1}{ }^{2}}\right. \\
\left.-\frac{-\exp \left(-\lambda_{2} t\right)+1}{\lambda_{2}}-\frac{\exp \left(-\lambda_{1} t\right)-1}{\lambda_{1}}\right) .
\end{gathered}
$$

We now solve the initial value problem given in the first line of (23) by the method of separation of variables. To do this we let $\eta_{0}(x, t)=X(x) T(t)$. Then, we get the following boundary value problem (BVP):

$$
X^{\prime \prime}(x)+\lambda X(x)=0, X^{\prime}(0)=X^{\prime}(1)=0 .
$$

If $\lambda=0$ we have $X_{0}(x)=B_{0}$. If $\lambda>0$ we have $\lambda_{n}=n^{2} \pi^{2}, \mathrm{n}=1,2, \ldots$ with $X_{n}(x)=B_{n} \cos (n \pi x)$. It is clear that the above $\mathrm{BVP}$ does not have any negative eigenvalues. Also, solving $T_{n}^{\prime}(t)+\lambda_{n} D_{1} T_{n}(t)=0$ yields $T_{0}(t)=C_{0}, T_{n}(t)=C_{n} \exp \left(-n^{2} \pi^{2} D_{1} t\right)$. Therefore, the solution to the problem becomes

$$
\eta_{0}(x, t)=B^{*}+\sum_{n=1}^{\infty} B_{n}{ }^{*} \exp \left(-n^{2} \pi^{2} D_{1} t\right) \cos (n \pi x)
$$

where

$$
B^{*}=\int_{0}^{1} \eta_{0}(x, 0) d x=1 \quad \text { and } \quad B_{n}^{*}=2 \int_{0}^{1} \eta_{0}(x, 0) \cos (n \pi x) d x=0(n=1,2, \ldots),
$$

which gives us that the solution is $\eta_{0}(x, t)=1$. Let us now solve the second equation in (23). If we write A, $A_{x}, \eta_{0}$ and $\eta_{0 x}$ in the second equation of (23), the equation becomes

$$
\eta_{1 t}=D_{1}\left(\eta_{1_{x x}}-6\left(6 x^{2}-6 x+1\right)\right)
$$

Then, we choose $v(x, t)=\eta_{1}(x, t)-3\left(x^{2}-2 x^{3}+x^{4}\right)$ to make the last equation homogeneous. Therefore, we obtain the following initial- boundary value problem:

$$
\begin{gathered}
v_{t}=D_{1} v_{x x}, \\
v(x, 0)=-3\left(x^{2}-2 x^{3}+x^{4}\right), \quad 0<x<1
\end{gathered}
$$




$$
v_{x}(0, t)=v_{x}(1, t)=0, t>0 .
$$

If we solve this problem we get

$$
v(x, t)=-\frac{1}{10}+\frac{9}{\pi^{4}} \sum_{n=1}^{\infty} \frac{\exp \left(-4 n^{2} \pi^{2} D_{1} t\right)}{n^{4}} \cos (2 n \pi x),
$$

and therefore we obtain the following

$$
\eta_{1}(x, t)=-\frac{1}{10}+\frac{9}{\pi^{4}} \sum \infty_{n=1} \frac{\exp \left(-4 n^{2} \pi^{2} D_{1} t\right)}{n^{4}} \cos (2 n \pi x)+3\left(x^{2}-2 x^{3}+x^{4}\right), \quad 0 \leq x \leq 1, t>0 .
$$

Thus, regular perturbation solutions of equation (23) become:

$$
\left\{\begin{array}{l}
\eta_{0}(x, t)=1 \\
\eta_{1}(x, t)=3\left(x-x^{2}\right)^{2}-\frac{1}{10}+\frac{9}{\pi^{4}} \sum_{n=1}^{\infty} \frac{\exp \left(-4 n^{2} \pi^{2} D_{1} t\right)}{n^{4}} \cos (2 n \pi x) .
\end{array}\right.
$$

Similarly, we can solve equations (24) and (25), respectively, and get the following solutions:

$$
\left\{\begin{array}{l}
\sigma_{0}(x, t)=1 \\
\sigma_{1}(x, t)=3\left(x-x^{2}\right)^{2}-\frac{1}{10}+\frac{9}{\pi^{4}} \sum_{n=1}^{\infty} \frac{\exp \left(-4 n^{2} \pi^{2} D_{2} t\right)}{n^{4}} \cos (2 n \pi x),
\end{array}\right.
$$

and

$$
\left\{\begin{array}{l}
m_{0}(x, t)=1, \\
m_{1}(x, t)=3\left(x-x^{2}\right)^{2}-\frac{1}{10}+\frac{9}{\pi^{4}} \sum_{n=1}^{\infty} \frac{\exp \left(-4 n^{2} \pi^{2} D_{3} t\right)}{n^{4}} \cos (2 n \pi x) .
\end{array}\right.
$$

As a result, the two-terms regular perturbation solutions of equations (19)-(25) are obtained as follows:

$$
\begin{gathered}
u(x, t, \varepsilon)=\theta(x) \exp \left(-\lambda_{2} t\right)-\varepsilon\left(\lambda_{2} t \theta(x) \exp \left(-\lambda_{2} t\right)\right)+O\left(\varepsilon^{2}\right), \\
v(x, t, \varepsilon)=\frac{\lambda_{2}}{\lambda_{1}-\lambda_{2}} \theta(x)\left(\exp \left(-\lambda_{2} t\right)-\exp \left(-\lambda_{1} t\right)\right)+\varepsilon \lambda_{2}^{2} \theta(x)\left(\frac{-t \exp \left(-\lambda_{2} t\right)}{\lambda_{1}-\lambda_{2}}+\frac{\exp \left(-\lambda_{2} t\right)-1}{\left(\lambda_{1}-\lambda_{2}\right)^{2}}\right) \\
-\varepsilon \frac{\lambda_{1} \lambda_{2}}{\lambda_{1}-\lambda_{2}} \theta(x)\left(\frac{\exp \left(-\lambda_{2} t\right)-1}{\lambda_{1}-\lambda_{2}}-\operatorname{texp}\left(-\lambda_{1} t\right)\right)+O\left(\varepsilon^{2}\right), \\
c(x, t, \varepsilon)=\frac{\lambda_{1} \lambda_{2}}{\lambda_{1}-\lambda_{2}} \theta(x)\left(\frac{1-\exp \left(-\lambda_{2} t\right)}{\lambda_{2}}+\frac{\exp \left(-\lambda_{1} t\right)-1}{\lambda_{1}}\right) \\
+\varepsilon \lambda_{1} \lambda_{2}^{2} \theta(x)\left(\frac{\exp \left(-\lambda_{2} t\right)}{\lambda_{2}\left(\lambda_{1}-\lambda_{2}\right)}-\frac{\exp \left(-\lambda_{2} t\right)+1}{\lambda_{2}\left(\lambda_{1}-\lambda_{2}\right)^{2}}-\frac{t}{\left(\lambda_{1}-\lambda_{2}\right)^{2}}\right) \\
-\varepsilon \frac{\lambda_{1} \lambda_{2}}{\lambda_{1}-\lambda_{2}} \theta(x)\left(-\frac{\exp \left(-\lambda_{2} t\right)+1+t}{\lambda_{1}-\lambda_{2}}+\frac{t \exp \left(-\lambda_{1} t\right)-\exp \left(-\lambda_{1} t\right)-1}{\lambda_{1}}\right. \\
\left.-\frac{\exp \left(-\lambda_{1} t\right)+1}{\lambda_{1}^{2}}+\frac{\exp \left(-\lambda_{2} t\right)+1}{\lambda_{2}}\right)+O\left(\varepsilon^{2}\right),
\end{gathered}
$$




$$
\begin{aligned}
& f(x, t, \varepsilon)=-\frac{\lambda_{1} \lambda_{2} \lambda_{4}}{\lambda_{1}-\lambda_{2}} \theta(x)\left(\frac{t}{\lambda_{2}}+\frac{\exp \left(-\lambda_{2} t\right)-1}{\lambda_{2}{ }^{2}}+\frac{-\exp \left(-\lambda_{1} t\right)+1}{\lambda_{1}{ }^{2}}-\frac{t}{\lambda_{1}}\right) \\
&-\varepsilon \frac{\lambda_{1} \lambda_{2} \lambda_{4} \beta}{\lambda_{1}-\lambda_{2}} \theta(x)\left(\frac{t^{2}}{2 \lambda_{2}}+\frac{-\exp \left(-\lambda_{2} t\right)+1}{\lambda_{2}{ }^{3}}-\frac{t}{\lambda_{2}{ }^{2}}+\frac{\exp \left(-\lambda_{1} t\right)-1}{\lambda_{1}{ }^{3}}-\frac{t}{\lambda_{1}{ }^{2}}\right) \\
&-\varepsilon \lambda_{1} \lambda_{2}{ }^{2} \lambda_{4} \theta(x)\left(\frac{\lambda_{2} t \exp \left(-\lambda_{2} t\right)+\exp \left(-\lambda_{2} t\right)-1}{\lambda_{2}{ }^{2}\left(\lambda_{1}-\lambda_{2}\right)}+\frac{-\exp \left(-\lambda_{2} t\right)+1}{\lambda_{2}\left(\lambda_{1}-\lambda_{2}\right)^{2}}-\frac{t}{\left(\lambda_{1}-\lambda_{2}\right)^{2}}\right) \\
&-\varepsilon \frac{\lambda_{1} \lambda_{2} \lambda_{4}}{\lambda_{1}-\lambda_{2}} \theta(x)\left(\frac{-\exp \left(-\lambda_{2} t\right)+1-\lambda_{2} t}{\lambda_{2}\left(\lambda_{1}-\lambda_{2}\right)}+\frac{\exp \left(-\lambda_{1} t\right)}{\lambda_{1}}+\frac{\exp \left(-\lambda_{1} t\right)-1}{\lambda_{1}{ }^{2}}\right. \\
&\left.-\frac{-\exp \left(-\lambda_{2} t\right)+1}{\lambda_{2}}-\frac{\exp \left(-\lambda_{1} t\right)-1}{\lambda_{1}}\right)+O\left(\varepsilon^{2}\right), \\
& \eta(x, t, \varepsilon)=1+\varepsilon\left(3\left(x-x^{2}\right)^{2}-\frac{1}{10}+\frac{9}{\pi^{4}} \sum_{n=1}^{\infty} \frac{\exp \left(-4 n^{2} \pi^{2} D_{1} t\right)}{n^{4}} \cos (2 n \pi x)\right)+O\left(\varepsilon^{2}\right), \\
& \sigma(x, t, \varepsilon)=1+\varepsilon\left(3\left(x-x^{2}\right)^{2}-\frac{1}{10}+\frac{9}{\pi^{4}} \sum_{n=1}^{\infty} \frac{\exp \left(-4 n^{2} \pi^{2} D_{2} t\right)}{n^{4}} \cos (2 n \pi x)\right)+O\left(\varepsilon^{2}\right), \\
& m(x, t, \varepsilon)=1+\varepsilon\left(3\left(x-x^{2}\right)^{2}-\frac{1}{10}+\frac{9}{\pi^{4}} \sum_{n=1}^{\infty} \frac{\exp \left(-4 n^{2} \pi^{2} D_{3} t\right)}{n^{4}} \cos (2 n \pi x)\right)+O\left(\varepsilon^{2}\right) .
\end{aligned}
$$

\section{Numerical example}

The figures below have been generated by using MATLAB with the parameter values $\varepsilon=0.01, D_{1}=0.25, D_{2}=0.45$, $\mathrm{D}_{3}=0.75, \lambda_{1}=8, \lambda_{2}=7.2, \lambda_{4}=10, \quad$ and $\beta=0.22$ for the numerical purpose. Figures (2)-(5) show the chemotactic agent, angiogenic factor, enzyme, and fibronectin densities, while Figures (6)-(8) show the endothelial, pericyte and macrophage cell densities, respectively. To obtain the numerical solutions of the cell equations we have used only four terms of the series given by the equations (37)-(39).

Numerical calculations of the equations (15)-(17) together with the initial and boundary conditions can be found in [1]. In this paper we obtain the regular perturbation solutions of the same equations and compare our results with those obtained in [1].

\section{Conclusions}

In this paper we have obtained regular perturbation solutions of a mathematical model for capillary formation in tumor angiogenesis. In [20] and [21] the one dimensional (where only endothelial cell equation has been considered) and the two dimensional versions of the model have been solved, respectively, by initial data perturbation approximation.

However, in [1] the one dimensional version of the model has been solved numerically by a classical explicit method 
which takes too much time to get the solution. We believe that the regular perturbation method is much more easier, effective and faster. As the reader remembers, we have used only four term expansion of the series in (37)-(39) to draw Figures (6)-(8). Even with four term expansion of the series solutions we have obtained what we expect to see for the endothelial, pericyte, macrophage cell movements in a capillary. This shows the effectiveness of our method. Of course, a more stable solution for the cell equations can be obtained by getting more terms of the series solution.

Our computations show that we get numerical instability of our solutions if we take $\varepsilon$ bigger than 0.1 . For example, the solutions blow up in finite time if we take $\varepsilon=0.15$. Of course, $\varepsilon$ sensitivity analysis could be made to determine which $\varepsilon$ is the best for our perturbation series approximation. We did not do this in this paper. We just picked some $\varepsilon$ to get the best approximation to the numerical solutions given in [1]. Also, we tried to take the diffusion constants $\mathrm{D}_{1}, \mathrm{D}_{2}$ and $\mathrm{D}_{3}$ within the same order of magnitude. They just differ by a constant. This is because of the biological facts about the diffusions of the cells.

We must also mention the importance of the choice of the function $\theta(\mathrm{x})$ appearing in the initial data. It is the function that initiates the dynamics in the model equations. If it is zero, all of the variables in the model stay dormant, and no action begins in the ECM for angiogenesis to occur.

Our computations were run from $\mathrm{t}=0$ until $\mathrm{t}=0.1$. This is because angiogenesis was completed by then [26]. Figure 2 is generated from equation (33). It shows the chemotactic agent present in the system. It is converted to the tumor angiogenic factor by the receptor on the $\mathrm{MC}$ wall. The resulting tumor angiogenic factor is obtained from equation (34) for the same time range, and it is presented in Figure (3). The amount of the macrophage cell (MC) during this reaction is obtained from equation (39), and it is shown in Figure (8). Similarly the amounts of the EC and PC present in the system (obtained from equations (37), (38), respectively) are shown in Figures (6) and (7). In Figure (4) the enzyme in the system is shown and it is obtained from equation (35). This enzyme is produced as a consequence of the reaction in equation (2). In Figure 5 the decay of the fibronectin is presented and it is obtained from equation (36). In this figure we see the advancing channel in the ECM.

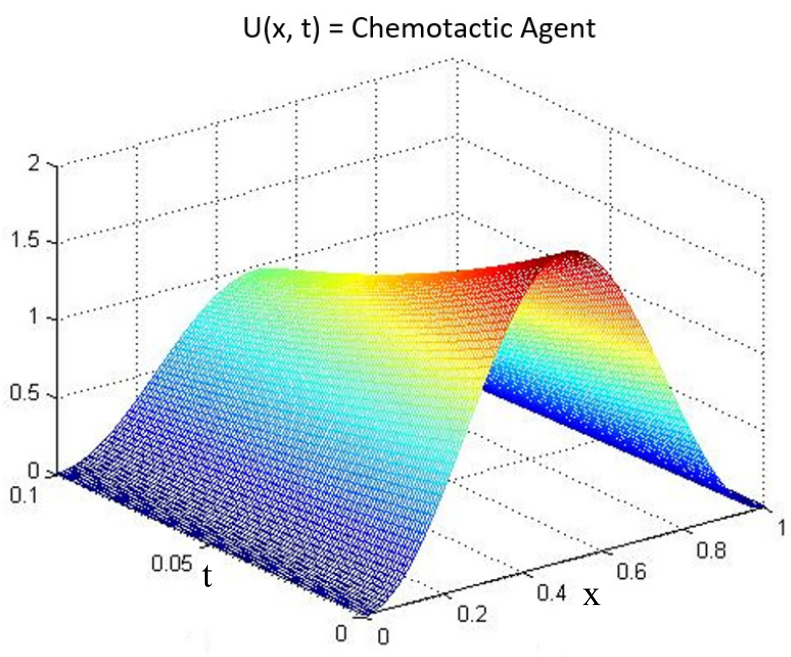

Fig. 2: Chemotactic Agent.

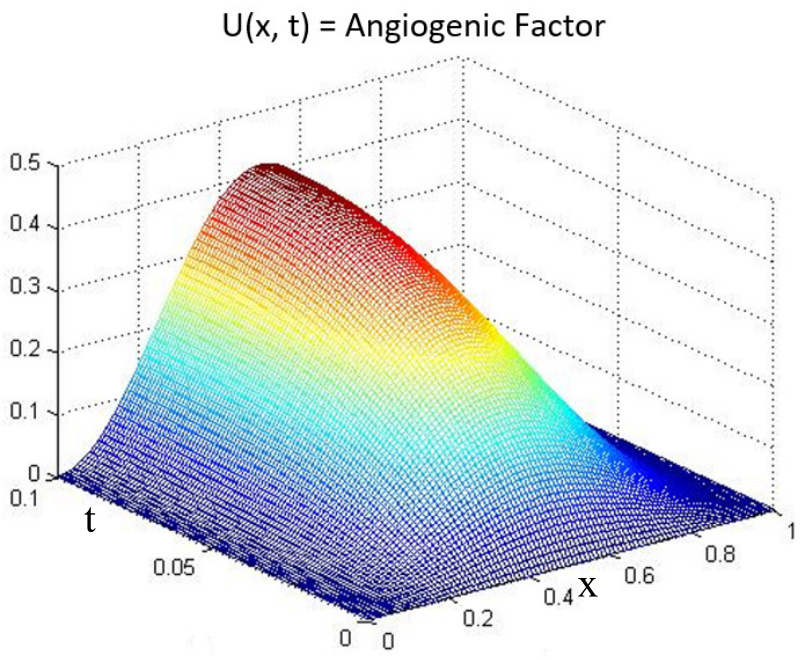

Fig. 3: Angiogenic Factor. 


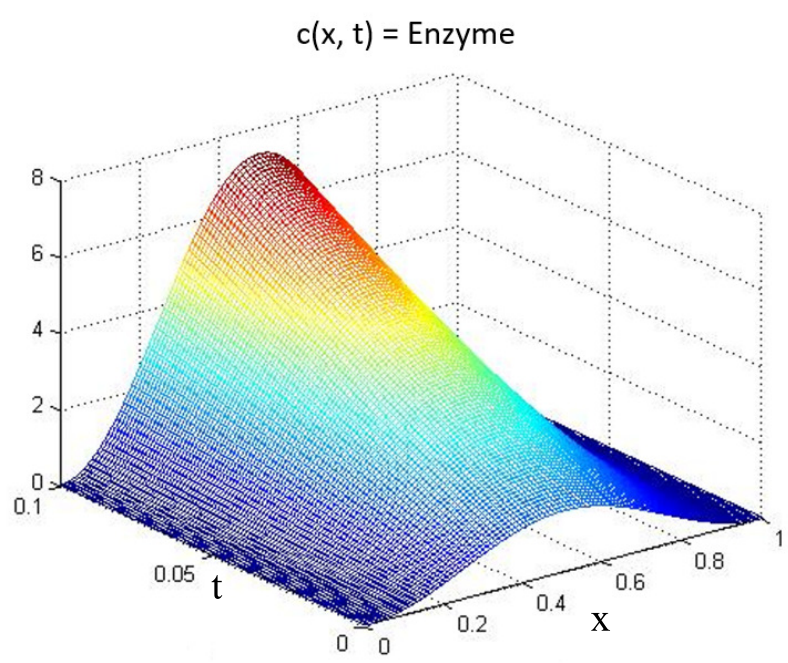

Fig. 4: Enzyme.

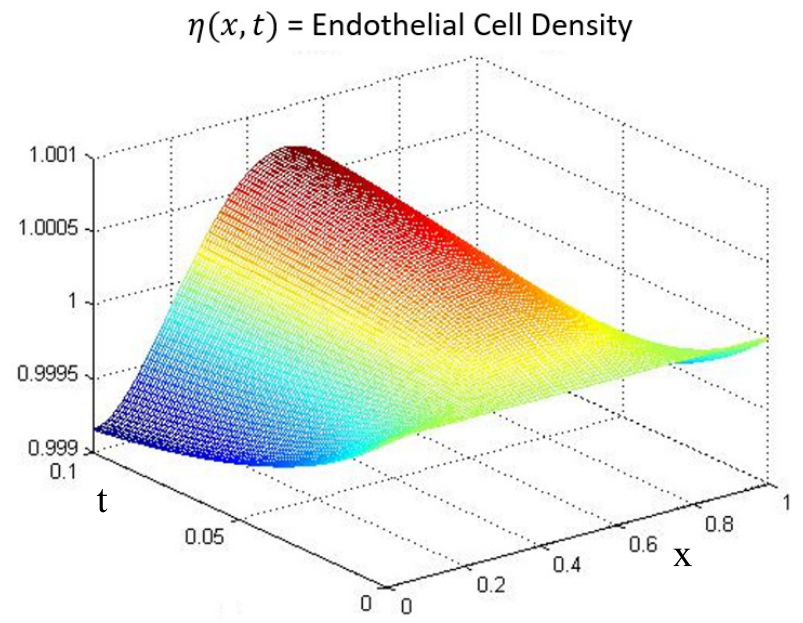

Fig. 6: Endothelial Cell Density.

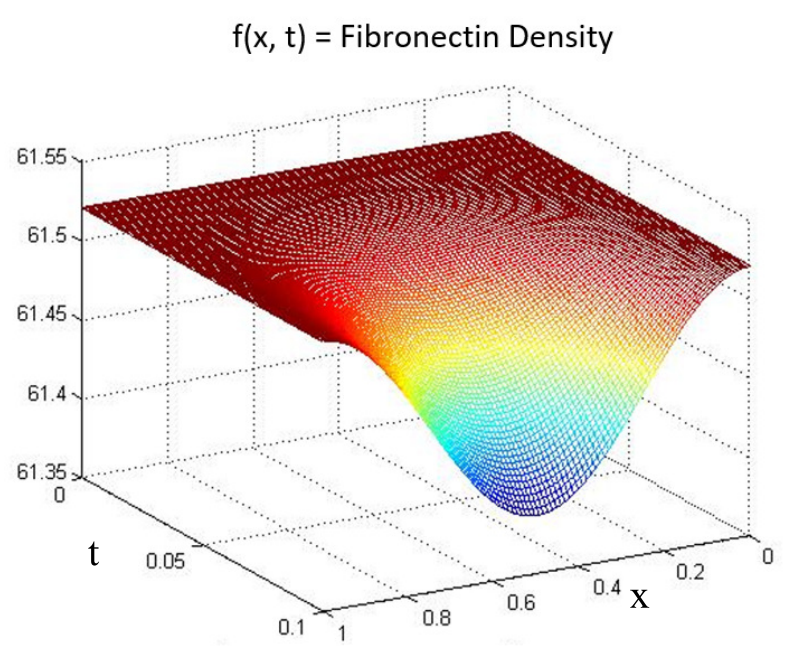

Fig. 5: Fibronectin Density.

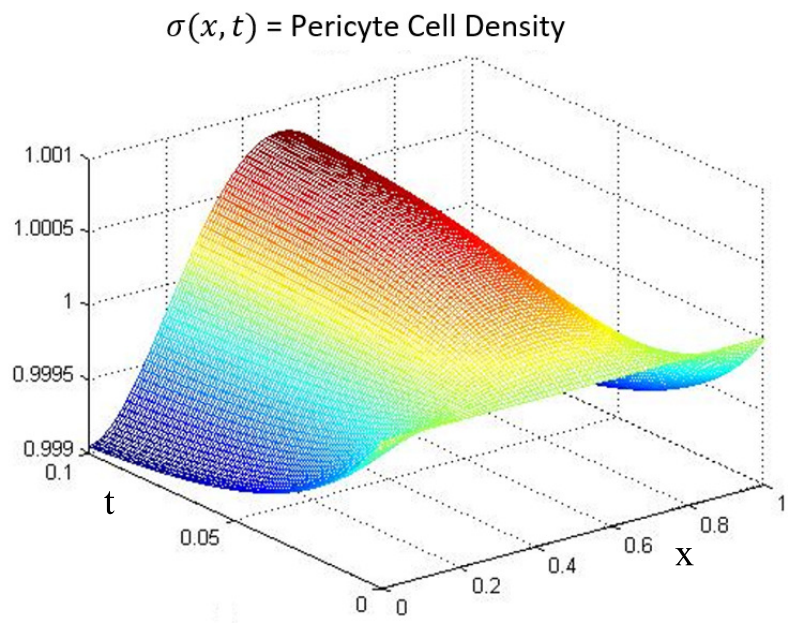

Fig. 7: Pericyte Cell Density.

\section{Competing interests}

The authors declare that they have no competing interests.

\section{Authors' contributions}

All authors have contributed to all parts of the article. All authors read and approved the final manuscript.

\section{References}

[1] Levine HA, Sleeman BD and Nilsen-Hamilton M. A mathematical model for the roles of pericytes and macrophages in the initiation of angiogenesis. I. The role of protease inhibitors in preventing angiogenesis (2000) Math Biosci 168: 77-115. https://doi.org/10.1016/s0025-5564(00)00034-1. 


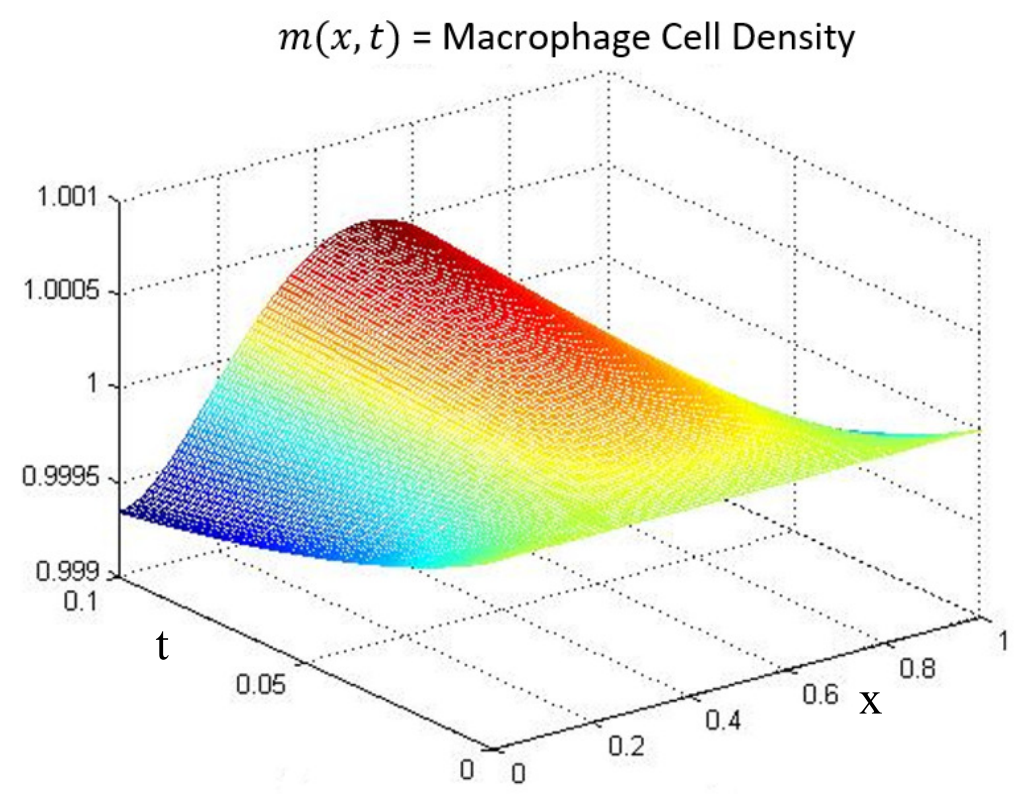

Fig. 8: Macrophage Cell Density

[2] Paweletz N and Knierim M. Tumor-related angiogenesis (1989) Crit Rev in Oncology/Hematology 9: 197-242.

[3] Nicosia RF, Bonanno E and Smith M. Fibronectin promotes the elongation of micro vessels during angiogenesis in vitro (1993) J Cell Phys 154(3): 654-661. https://doi.org/10.1002/jcp.1041540325.

[4] Yamada KM and Olden K. Fibronectins-adhesive glycoproteins of cell surface and blood (1978) Nature, 275: 179-184. https://doi.org/10.1038/275179a0.

[5] Folkman J. Tumor angiogenesis: therapeutic implications (1971) N Engl J Med 285: 1182-1186. http://dx.doi.org/10.1056/NEJM197111182852108.

[6] Folkman J. The vascularization of tumors (1976) Sci Amer 234: 58-70.

[7] Schleef RR and Birdwell CR. The effect of proteases on endothelial cell migration in vitro (1982) Exp Cell Res 141: 505-508. https://doi.org/10.1016/0014-4827(82)90244-0.

[8] Othmer HG and Stevens A. Aggregation, blow up, and collapse: the ABCS of taxis in reinforced random walks (1997) SIAM J Appl Math 57: 1044-1081. https://epubs.siam.org/doi/10.1137/S0036139995288976.

[9] Pamuk S. Qualitative analysis of a mathematical model for capillary formation in tumor angiogenesis (2003) Math Mod and Meth in Appl Sci 13: 19-33.

[10] Schor AM and Schor SL. Tumour angiogenesis (1983) J Path, 141: 385-413.

[11] Bellomo N, Li NK and Maini PK. On the foundations of cancer modelling: selected topics, speculations, and perspectives (2008) Math Mod and Meth in Appl Sci, 18: 593-646.

[12] Bellomo N, Bellouquid A, Nieto J and Soler J. On the asymptotic theory from microscopic to macroscopic growing tissue models: an overview with perspectives (2012) Math Mod and Meth in Appl Sci, 22: Article ID1130001. https://doi.org/10.1142/S0218202512005885.

[13] Bellouquid A and De Angelis E. From kinetic models of multicellular growing system stoma macroscopic biological tissue models (2011) Nonlinear Anal Real World App 12: 1111-1122. https://doi.org/10.1016/j.nonrwa.2010.09.005.

[14] Zheng Z, Koh GY and Jackson T. A continuous model of angiogenesis initiation, extention, and maturation of new blood vessels modulated by vascular endothelial growth factor, angiopoietins, platelet-derived growth factor-b and Pericites (2013) Discrete and Cont Dyn S 18: 1109-1154. https://doi.org/10.3934/dcdsb.2013.18.1109.

[15] Mantzaris NV, Webb S and Othmer HG. Mathematical modeling of tumor-induced angiogenesis (2004) J Math Biol 49: 111-187. https://doi.org/10.1007/s00285-003-0262-2. 
[16] Radhika TSL, Iyengar TKV and Raja Rani T. Approximate Analytical Methods for Solving Ordinary Differential Equations (2014) A Chapman \& Hall Book / CRC.

[17] Bender CM and Orszag SA. Advanced Mathematical Methods for Scientists and Engineers (1999) Springer New York.

[18] Levine HA, Pamuk S, Sleeman BD and Nilsen-Hamilton M. Mathematical modeling of capillary formation and development in tumor angiogenesis: Penetration into the stroma (2001) Bull Math Biol 63: 801-863. https://doi.org/10.1006/bulm.2001.0240

[19] Davis B. Reinforced random walk (1990) Probab Th Rel Fields 84: 203-229. https://doi.org/10.1007/BF01197845.

[20] Pamuk S. Solution of a Linearized Mathematical Model for Capillary Formation in Tumor Angiogenesis: An Initial Data Perturbation Approximation (2013) Comput Math Methods Med 2013: Article ID 789402. http://dx.doi.org/10.1155/2013/789402.

[21] Pamuk S and Çay I. A mathematical analysis of a 2D model for tumor angiogenesis: An initial data perturbation approximation (2018) Communication in Math Modeling Appl 3: 13-27.

[22] Pamuk S and Bajjah B. Steady- state analysis of a two dimensional model for tumor angiogenesis in the absence of endothelial cell proliferation (2016) Academic J Appl Math Sci 2: 102-108.

[23] Roberts SM. Variational perturbation method and power-series approximation method (1980) J Optimization Theory and Appl 32: 441-450. https://doi.org/10.1007/BF00934032.

[24] Shivamoggi B. Perturbation Methods for Differential Equations (2003) Springer Science + Business Media New York.

[25] Zwillinger D. Handbook of Differential Equations (1997) 3rd edition Academic Press.

[26] Pamuk S, Çay I, Sazcı A. A 2D mathematical model for tumor angiogenesis: The roles of certain cells in the extra cellular matrix (2018) Math Biosci 306: 32-48. https://doi.org/10.1016/j.mbs.2018.10.007.

[27] Eldesoky IM, Abdelsalam SI, El-Askary WA and Ahmed MM. Concurrent development of thermal energy with magnetic field on a particle-fluid suspension through a porous conduit (2019) BioNanosci 9: 186-202. https://doi.org/10.1007/s12668-018-0585-5.

[28] Koumy SR, Barakat EI and Abdelsalam SI. Hall and porous boundaries effects on peristaltic transport through porous medium of a Maxwell model (2012) Transp Porous Med 94: 643-658. https://doi.org/10.1007/s11242-012-0016-y.

[29] Abdelsalam SI and Vafai K. Combined effects of magnetic field and rheological properties on the peristaltic flow of a compressible fluid in a microfluidic channel (2017) Eur J Mech B/Fluids 65: 398-411. https://doi.org/10.1016 /j.euromechflu. 2017.02.002.

[30] Abdelsalam SI and Vafai K. Particulate suspension effect on peristaltically induced unsteady pulsatile flow in a narrow artery: Blood flow model (2017) Math Biosci 283: 91-105. https://doi.org/10.1016/j.mbs.2016.11.012.

[31] Mekheimer KS, Komy SR and Abdelsalam SI. Simultaneous effects of magnetic field and space porosity on compressible Maxwell fluid transport induced by a surface acoustic wave in a microchannel (2013) Chin Phys B 22: 124702. http://dx.doi.org/10.1088/1674-1056/22/12/124702. 